\title{
FOCUS ON CANCER PROTEOMICS
}

\section{Functional proteomics to dissect tyrosine kinase signalling pathways in}

\section{cancer}

Walter Kolch ${ }^{1,2}$ \& Andrew Pitt ${ }^{3}$

${ }^{1}$ Conway Institute, University College Dublin, Belfield, Dublin 4, Ireland

${ }^{2}$ Systems Biology Ireland, University College Dublin, Belfield, Dublin 4, Ireland

${ }^{3}$ Sir Henry Wellcome Functional Genomics Facility, University of Glasgow, Glasgow G12 8QQ, UK Correspondence to WK: walter.kolch@ucd.ie

\begin{abstract}
Advances in the generation and interpretation of proteomics data have spurred a transition from focussing on protein identification to functional analysis. Here, we review recent proteomics results that have elucidated new aspects of the role and regulation of signal transduction pathways in cancer using the epidermal growth factor receptor (EGFR), ERK and breakpoint cluster region (BCR)-ABL1 networks as examples. The emerging theme to understand cancer signalling as networks of multi-protein machines that process information in a highly dynamic environment that is shaped by changing protein interactions and posttranslational modifications (PTMs). Cancerous genetic mutations derange these protein networks in complex ways that are tractable by proteomics.
\end{abstract}


While the causes of cancer lie in mutations or epigenetic changes at the genetic level, their molecular manifestation is the dysfunction of biochemical pathways at the protein level. Therefore, it is only by studying the proteome that we can extract functional understanding of the pathways that are deranged in cancer. Rather than just identifying proteins, functional proteomics focuses on the generation of information about proteins, such as expression levels, PTMs and activity, which directly contribute to a functional understanding of the biological system. Functional proteomics feeds directly into the systematic analysis of biochemical networks, often using mathematical modelling or other systems biology tools. It also provides readouts for chemical biology and chemical genetics necessary to interpret the action of drugs.

The growing interest in functional proteomics is not only fuelled by the prospect of a true functional understanding, but also by significant improvements in technology and methodology. Advances in mass spectrometry (MS) have extended the sensitivity, accuracy and speed of analysis to now routinely enable the identification of several thousand proteins per experiment. The introduction of MS methods for accurate relative and absolute protein quantification, and the large scale analysis of PTMs, such as phosphorylation and ubiquitinylation, has enabled truly functional proteomics. MS is now joined by antibody and protein-protein interaction arrays ${ }^{1}$, fluorescence and flow cytometry based detection of proteins and $\mathrm{PTMs}^{2}$, and optical spectroscopic methods of proteome analysis ${ }^{3,4}$. These latter techniques are promoted by an ever increasing repertoire of specific antibodies against proteins and PTMs, and bring single cell proteomics into reach. 
Proteomics has been widely applied to cancer research and the mapping of cancerous signalling pathways, and the vast literature exceeds the scope of a single review. Therefore, we will use three main cancer pathways, EGFR, BCR-ABL1 and ERK as examples to elaborate some of the new paradigms in cancer signalling that owe their discovery to proteomics. The EGFR network is frequently altered in various human cancers, and has been extensively studied using proteomics both at the level of the receptor and the downstream pathways. BCR-ABL1 stands out amongst oncogenes as chronic myelogenous leukaemia (CML), which is caused by this fusion gene, is with few exceptions strictly dependent on BCR-ABL1 function ${ }^{5}$. Thus, BCR-ABL1 signalling could reveal the minimum assortment of pathways required for transforming a human cell. The ERK pathway is a critical effector of both the EGFR and BCR-ABL1 pathways. Hence, although other important pathways - such as the AKT or mTOR pathways - are not discussed here, our selection allows us to elaborate general principles and representative new insights into cancer signalling pathways that we have gained through proteomics. As technological and methodological advances are integral to functional proteomics, we briefly discuss the experimental approaches, although the emphasis is on the biological findings.

We begin with discussing protein-protein interactions. Most cellular processes, including signal transduction, require the coordinated formation of multiprotein complexes. A main focus in functional proteomics has been to analyse the formation of specific protein-protein interactions and the consequent assembly of macromolecular protein complexes, in particular how these assemblies are regulated by scaffolding proteins and PTMs, and how they affect pathway function. We then 
discuss selected PTMs that are associated with cancer pathways. While phosphorylation and glycosylation have dominated the post-translational area, we increasingly realise that a large range of modifications, including ubiquitinylation, play critical roles in cellular regulation. We conclude with an outlook towards single cell proteomics and clinical relevance of functional cancer proteomics.

\section{Protein-protein interactions}

Protein-protein interactions play important roles in cellular processes. However, deconvoluting the complex nature of these interactions has proved to be a difficult undertaking. Stable protein assemblies function as 'molecular machines' in all cellular processes from transcription machineries in the nucleus, to ribosomes that translate mRNA into proteins and molecular motors that generate the force for intracellular transport and cell motility. These stable protein complexes are highly amenable to proteomic analysis, and powerful isolation procedures have been developed to purify stable protein complexes for MS analysis. They usually employ tandem affinity purification (TAP)tags, which allow the efficient purification of stable protein complexes by a sequential pulldownelution-pulldown protocol $^{6}$. Genome wide interaction studies ${ }^{7,8}$ using this methodology showed that the yeast proteome is organized into 500-550 protein complexes, thus experimentally confirming the concept that organization based on protein-protein interactions can specify biological functions. Does this concept also apply to molecular information processors in mammalian cells? 
In principle, dynamically changing protein interactions could provide the speed, plasticity and compartmentalization needed to process signals that encode both temporal and spatial information. However, these associations may be fleetingly transient, featuring complex dynamics and stoichiometries between different complexes competing for the same component. Thus, these interactions are often regulated through transient PTMs, such as phosphorylation. Specific phosphorylation-dependent binding motifs have been identified on many signalling proteins. For example, activated receptor tyrosine kinases (RTKs) phosphorylate tyrosines in their cytoplasmic kinase domains that provide binding sites for downstream effectors that direct the assembly of transient signalling complexes ${ }^{9-11}$. RTKs, especially those of the ErbB family, are often overexpressed or mutated in cancer, resulting in the assembly of functionally aberrant signalling complexes ${ }^{9,12}$.

The technical aspects of mapping protein interactions in signalling pathways were covered in recent reviews ${ }^{13-15}$. The methodologies rely on the application of quantitative mass spectrometric techniques $^{16}$ (Box 1) and often require the generation of dynamic interaction maps ${ }^{17,18}$. One of the main advantages is that the quantitative techniques can separate true interactors from contaminations ${ }^{19}$. However, significant challenges remain. Methodologies for measuring the specific occupancy of PTM sites on individual protein molecules are limited. Similarly, we cannot resolve the specific functions of individual proteins within different complexes, which is akin to having a corporate organization chart with all the names but no affiliations to departments. However, emerging evidence suggests that signal transduction uses a matrix management approach, 
where one component fulfils different tasks in different complexes (or departments). Mapping this matrix is further complicated by the fact that the structure can change dynamically over time, and certain complexes may only be necessary for a limited time in order to activate their downstream biological pathways.

This provides a major challenge, as it may be failures in the matrix management that drive cancer rather than individual mutations altering one function. The crosstalk between protein complexes also contributes to the robustness of cancer signalling by providing modularization ${ }^{20}$ and generating molecular heterogeneity ${ }^{21}$ that together allow rapid and versatile adaptation. This view is encapsulated in the concept of non-oncogene addiction, which highlights the importance of normal pathways supporting the biological effects of mutated pathways ${ }^{22}$. This concept has wide ramifications for the design of rational cancer therapies and the identification of promising targets. The hope is that the common phenomenon of cancer drug resistance can be conquered by the parallel targeting of oncogenic pathways and non-oncogene addictive alterations.

Protein complexes. The EGFR pathway is one of the most extensively studied signalling pathways that is relevant to cancer ${ }^{23}$ and the EGFR interactome is one of the most well described both biochemically ${ }^{24}$ and theoretically ${ }^{25,26}$ (Fig. 1). A major question is how EGFR coordinates diverse biological responses and how response specificity is generated. Functional proteomics has deciphered the components of protein complexes formed by EGFR and has also contributed to an understanding of the topological organisation the downstream signalling pathways. 
Using MS-based quantitative proteomics to analyse proteins interacting with phosphopeptides that are phosphorylated in response to ligand binding showed that the four members of the ErbB family (EGFR is also known as ERBB1) have differential preferences for interaction partners ${ }^{27}$. Interestingly, growth factor receptor-bound protein 2 (GRB2), which initiates activation of the ERK pathway, and PI3K, which activates the AKT pathway, have several binding sites on one receptor. Multimeric binding could be a simple mechanism to transmit strength of the input into these pathways. A complementary approach used protein microarrays comprising most known SH2 and phosphotyrosine binding (PTB) domains in the human genome to measure the dissociation constants of each domain for 61 peptides that are physiologically tyrosine phosphorylated when ERBB1-4 are activated ${ }^{28}$. The resulting systematic interaction maps showed that the binding complement differs between receptor dimers, with ERBB2-ERBB3 having the most interactions followed by EGFR-ERBB3>EGFR-ERBB2>EGFR-EGFR. This distribution correlates with the transforming potencies of ErbB dimers ${ }^{29,30}$ indicating that an increase in interaction partners enhances oncogenicity. Interestingly, EGFR and ERBB2 (also known as HER2), but not ERBB3, became increasingly promiscuous with increasing expression levels or activation state resulting in the binding of more proteins and the activation of more signalling pathways at higher concentration $s^{28}$, which may explain why they are often overexpressed in cancer. Comparing the interactors identified in these studies shows overlaps but also differences, for example signal transducer and activator of transcription 5 (STAT5) was only found to bind to EGFR and ERBB4 in 
the MS study. These differences could be due to different experimental systems or reflect differences in the technologies used.

These studies also suggested that the biological specificity of receptor signalling is determined by the composition of the signalling complexes they assemble; and the ligand induced changes in their interactomes. Implicit in this hypothesis is the assumption that changes in the receptor interactome are propagated throughout the network to affect downstream protein interactions and thereby dynamically shape the network topology used to transduce a signal. This theory was recently confirmed by the analysis of the dynamic ERK interactome in the control of differentiation versus proliferation in PC12 cells ${ }^{31}$. The ERK pathway consists of a three-tiered kinase cascade, Raf - MEK -ERK, and is a main effector of EGFR signalling (Fig. 1) that mediates many responses to EGF that are subverted in cancer, such as cell proliferation, transformation, differentiation, migration and survival. Stable isotope labelling with amino acids in culture (SILAC)-based quantitative proteomics was used to determine changes in the ERK interactome in response to nerve growth factor (NGF; which induces differentiation) and EGF (which induces proliferation). The results increased the number of proteins identified in the ERK interactome from 170 proteins $^{32}$ to $>280$. Importantly, 60 protein interactions changed in a differentiation-dependent manner. Detailed analysis of a subset of ERK interactors showed that they regulate the pathway at different steps, including the activation kinetics of ERK, crosstalk with other pathways and phosphorylation of transcription factors. These biochemical data were incorporated into a 
mathematical model revealing that the EGF and NGF pathways are under a distributed control mechanism rather than governed by a single master switch.

These results show that signalling pathways critically rely on protein interactions, which distribute control throughout the network, and that quantitative interaction proteomics is a formidable tool to map these pathways and their topologies. Furthermore, these findings predict that it is difficult to disrupt signalling networks by interventions targeting a single node, adding a mechanistic explanation to the clinical experience that the efficacy of single agent cancer therapies is usually limited. These studies are complemented by the analysis of downstream phosphorylation events that we discuss further below.

The proteomic studies of the EGFR pathway demonstrate how signalling specificity is achieved through regulated protein interactions, and how receptor overexpression can expand the scope of interaction partners sufficiently to drive oncogenesis. This theme of oncogenesis resulting from the unwanted spread of signalling into many pathways due to an expansion of the number of protein complexes assembled is more clearly demonstrated when this regulation is usurped by an oncoprotein. BCR-ABL1 is an oncogenic fusion protein that results from the chromosomal translocation that causes chronic myelogenous leukaemia (CML), and inhibition of its kinase activity can profoundly reverse the diseas ${ }^{33,34}$. The translocation that results in $B C R-A B L 1$ is a rare case where human tumorigenesis can be tied to a single genetic event both based on molecular evidence and the unprecedented clinical success of the BCR-ABL1 inhibitor imatinib ${ }^{5}$. 
What causes this exception? Classical biochemical studies suggested that BCR-ABL1 is a constitutively active tyrosine kinase and 'super-adaptor' that interacted with almost every pathway implicated in oncogenic transformation of haematopoietic cells and fibroblasts ${ }^{35}$ (Fig. 2). The challenge was to define which of these pathways is required for BCR-ABL1-induced transformation. An interaction proteome screen of BCR-ABL1-associated proteins revealed a network of several hundred proteins as direct or indirect interactors ${ }^{36}$. Evaluation of the highly connected hubs only revealed seven core components: GRB2, Src homology 2 domain containing transforming protein 1 (SHC1), CRK, CBL, p85 (also known as PIK3R2), suppressor of T-cell receptor signaling 1 (STS1, also known as UBASH3B) and SH2 domain-containing inositol phosphatase 2 (SHIP2, also known as INPPL1), which coordinate a signalling network comprising several hundred proteins (Fig. 2). Interestingly, many of the core components are scaffolds suggesting that BCR-ABL1 signalling is orchestrated through propagating layers of protein complexes. BCR-ABL1 kinase inhibitors disrupted this network leading the authors to conclude that the action of theses drugs should be considered as interfering with the equilibrium state of an intricate network of protein complexes rather than just inhibiting a single component. Given that several proteins interact with more than one of the seven core components, even the perturbation of a single interaction should have repercussions throughout the network as it could shift the equilibrium composition of other complexes. This view may become important in the quest for alternative strategies that can circumvent resistance to tyrosine kinase inhibitors. 
The big question is, of course, whether these findings from cultured cells translate into the clinic. This question was addressed mainly by using reverse phase protein microarrays, where lysates of tumour samples are arrayed and interrogated with antibodies. This strategy only permits targeted studies, but can examine large numbers of patients and functions with the limited amounts of tissue, such as tumour biopsies, that is typically available in clinical settings. Such studies have yielded clinically useful information and are discussed in detail by Brennan et al. in this issue ${ }^{37}$.

Protein Scaffolds. Having made the point that protein interactions are important, the question is what holds them together? Through binding two or more client proteins simultaneously scaffold proteins generate platforms that alter signalling kinetics, control crosstalk between signalling pathways and insulate elements from each other. Although the role of scaffolds in cancer still needs to be elaborated, we will briefly discuss them as they play fundamental roles in regulating both steady state and acute response kinetics of many pathways involved in cancer. They seem to be most relevant to mechanisms of non-oncogene addiction ${ }^{22}$, whereby normal components of signalling networks support the aberrant components. Aberrations in ERK signalling are common in cancer ${ }^{38}$ and hence we discuss two ERK pathway scaffolds.

The kinase suppressor of Ras (KSR) proteins scaffold the three-tiered Raf-MEK-ERK kinase module and contribute to the regulation of its signalling dynamics and spatio-temporal control. KSR1 is not essential for, but enhances ERK activation ${ }^{39}$. Importantly, knocking out KSR1 restrains transformation by oncogenic HRAS-G12V in cultured cells ${ }^{40}$ and HRAS-G12V driven skin 
carcinogenesis in mice ${ }^{41}$. KSR1 constitutively interacts with MEK and the complex translocates to the plasma membrane on mitogen stimulation ${ }^{42}$. MEK bound to KSR1 also promotes the recruitment of BRAF, which enhances MEK phosphorylation by BRAF. Interestingly, ERK binding induces feedback phosphorylation of KSR1 and BRAF by ERK, which promotes the dissociation of BRAF and KSR1 and release from the plasma membrane into the cytosol. It is unclear whether this is a purely negative feedback pathway that limits ERK activation, or whether this feedback also has a role in ensuring the turnover of clients on the scaffold and in localising the signal. In addition, KSR1 directly activates Raf kinases allosterically by forming side-to-side dimers ${ }^{43}$. KSR1 selectively interacts with ERK dimers and confines ERK signalling to cytosolic substrates and the expression of ERK point mutants that prevent its dimerization counteracts cellular transformation in vitro and tumour development in mouse xenograft models ${ }^{44}$. Thus, KSR1 can control several aspects of ERK signalling including substrate specificity and spatio-temporal activation kinetics.

Recently, the KSR2 interactome was studied using quantitative MS-based proteomics to compare KSR2 binders with and without tumour necrosis factor- $\alpha(\mathrm{TNF} \alpha)$ stimulation $^{45}$. Of $>100$ proteins potentially in the complex approximately 40 were recruited on TNF $\alpha$ stimulation, highlighting the highly dynamic properties of this signalling complex. Interestingly, KSR2 recruited ARAF rather than RAF1 or BRAF. ARAF is a poor activator of MEK, and its main, kinaseindependent, function is as an inhibitor of the pro-apoptotic mammalian STE20-like protein kinase 2 (MST2, also known as STK3) pathway ${ }^{46}$ indicating that KSR2 may have a role in redirecting ARAF to the ERK pathway. As scaffolding vastly accelerates reaction rates by co-localising enzyme and 
substrate $^{47}$, KSR2 bound ARAF is expected to be an effective MEK activator. Thus, this shift of ARAF from its anti-apoptotic function in the MST2 pathway to the mitogenic ERK pathway may contribute to oncogenic transformation.

A detailed comparison of the KSR1 and KSR2 interactomes showed the similarity of these scaffolds in promoting RTK-mediated ERK signalling, but also identified specific interactions. For example, the calcium dependent phosphatase calcineurin only binds to KSR2 ${ }^{48}$. In response to calcium signals calcineurin dephosphorylates the 14-3-3 binding sites on KSR2, which permits KSR2 membrane recruitment and activation of the ERK pathway ${ }^{48}$. Another proteomic study showed that KSR2 binds to AMP kinase (AMPK, also known as PRKAB1) mediating its stimulatory effects on glucose uptake and fatty acid oxidation ${ }^{49}$. This observation raises an interesting hypothesis: that KSR2 may coordinate links between mitogenic signalling and metabolic pathways by binding and coordinating the functions of ARAF and AMPK. ARAF can bind and inhibit pyruvate kinase M2 (PKM2) $)^{50}$. In many cancers PKM2 is aberrantly expressed in its low activity dimeric form, which favours aerobic glycolysis that is the hallmark of the Warburg effect ${ }^{51}$. Thus, KSR2 could enhance both AMPK mediated glucose uptake and PKM2 inhibition by ARAF, thereby promoting the Warburg effect and tumour growth. Consequently, KSR2 downregulation should ameliorate the Warburg effect and slow tumour growth, a prediction that is easily testable as $\mathrm{Ksr}^{-/-}$ mice are available ${ }^{49}$.

The above examples illustrate the key role of protein complexes in signalling, but equally highlight the importance of dynamic changes in the interactions that ensure the proper 
interpretation of the signal and the response to it. Conceptually, this view has significant implications. They include the notion that connections in signalling pathways are not hardwired, that a protein with a defined biochemical function can adopt different biological functionalities as part of different protein complexes, and that the kinetics of signalling specificity intimately involves dynamic changes in protein interactions. How are they brought about? Undoubtedly, PTMs are a salient part of the answer. The specific roles of PTMs in determining protein complex formation are just emerging, but many proteomic techniques for PTM analysis are available. In the following section we will briefly review how these technologies were applied to study of dynamic processes in cell signalling.

\section{PTMs}

PTMs are widely studied dynamic processes in cell signalling, which is not surprising considering the fundamental roles they play in regulating signalling pathways, and many studies have mapped cancer-associated PTMs. Phosphorylation of serine, threonine and tyrosine residues have received the most attention from the proteomics community, although ubiquitinylation, acylation and glycosylation are coming into the limelight. O-linked $\mathrm{N}$-acetylglucosamine acylation (OGlcNAcylation) is also receiving significant interest mainly due to its competing role in regulating phosphorylation by blocking potential phosphorylation sites.

Phosphorylation. Quantitative methods for the study of protein phosphorylation have been 
described elsewhere ${ }^{13,52-56}$. The first phosphoproteome to be extensively studied was that of EGFR activation (reviewed by Blagoev ${ }^{24}$ ), but several other RTK pathways have now been described.

Global tyrosine phosphorylation is usually mapped using enrichment with antiphosphotyrosine antibodies and subsequent MS analysis. Employing this method, both time course studies of EGF induced tyrosine phosphorylation and comparisons of signalling between different RTKs were performed ${ }^{57}$. For instance, SILAC based quantitative proteomics was used to compare tyrosine phosphorylation induced by EGF versus platelet derived growth factor (PDGF) in human mesenchymal stem cells ${ }^{58}$. EGF induces osteogenic differentiation while PDGF sustains proliferation and migration ${ }^{59}$. Most tyrosine phosphorylation events were common to either growth factor, except that the PI3K pathway was exclusively stimulated by PDGF. Inhibiting PI3K converted PDGF into a differentiation factor, elegantly demonstrating that biological specificity of RTK signalling is encoded by distinct biochemical differences in downstream signalling pathways arising from the protein complexes assembled at RTKs. A more recent study analysing tyrosine phosphorylation induced by EGF in human mammary epithelial cells revealed significantly different stoichiometries of phosphorylation at different sites within the same protein ${ }^{60}$ suggesting an intricate temporal and dynamic regulation.

This strategy was also successfully used to map the tyrosine phosphorylation network induced by cancer-associated EGFR mutants. Glioblastoma or astrocytoma grade IV, a highly malignant brain tumour, often expresses a truncated EGFR mutant, EGFRvIII, which is expressed from an amplified gene locus and enhances malignant behaviour in a dose dependent manner ${ }^{61}$. 
SILAC analysis of glioblastoma cell lines expressing different EGFRvIII levels and a kinase dead EGFRvIII as a control revealed that increasing EGFRvIII expression shifts signalling from ERK and STAT3 towards the PI3K pathway and also induce phosphorylation and transactivation of the hepatocyte growth factor receptor $\mathrm{MET}^{62}$. Consequently, combining EGF and MET inhibitors synergized to kill glioblastoma cells in vitro, producing a rational drug combination based on phosphoproteomics data.

Another proteomics study in non-small-cell lung cancer (NSCLC) cell lines compared phosphotyrosine signalling induced by wild-type EGFR, mutation-activated EGFR and overexpressed $\mathrm{MET}^{63}$. It revealed networks that extensively overlapped in the regulation of cell adhesion, motility, proliferation and survival. Interestingly, mutant EGFRs preferentially induced phosphorylation of cytoskeletal proteins, scaffolds implicated in cytoskeletal regulation and motility, as well as negative feedback inhibitors of EGFR signalling, such as sprouty 1 (SPRY1), SPRY2, SPRY4, and mitogen-inducible gene 6 (MIG6, also known as ERRFI1). The functional effects are largely unknown, but it is tempting to speculate that mutant EGFRs can sabotage the function of physiological feedback inhibitors. This hypothesis is consistent with observations that EGFR feedback inhibitors are often downregulated in cancers ${ }^{64}$. Crosstalk between MET and mutant EGFRs provided mutual activation of the respective receptors and downstream signalling pathways ${ }^{63}$. Interestingly, the sensitivity to EGFR and MET inhibitors as measured by biochemical outputs correlated with the extent of control of the respective receptors over the downstream network. This puts an interesting twist on the observation discussed above that EGFR oncogenicity 
increases with the number of pathways it engages ${ }^{28-30}$ as such functional expansion should also increase the vulnerability of the downstream network to receptor inhibition. The available clinical experience with EGFR inhibitors shows that spectacular regressions are achievable in patients with NSCLC, but this only occurs in a small subset of patients and without lasting effect ${ }^{65}$.

Does this reflect an arms race between increasing fragility and adaptive potential of RTK networks simultaneously afforded by the recruitment of an increasing number of downstream effectors? This hypothesis suggests the counterintuitive approach to combine EGFR inhibitors with inhibitors of downstream pathways in order to boost drug efficacy and prevent resistance. The combined inhibition of a receptor and a downstream effector could offer simple, general and hence attractive guidelines for the design of combination therapies and seems worthwhile exploring further.

A large scale phosphotyrosine proteomic screen using 41 NSCLC cell lines and >150 NSCLC tumours found a large number of RTKs, including EGFR and MET, and non-RTKs were activated ${ }^{66}$. The significance of most of these kinases for the pathogenesis of NSCLC is unknown, but an important prediction of this study is that the drug responsiveness of individual tumours should be highly dependent on whether these activated kinases function as part of hierarchical networks or in independent pathways. If they form hierarchical networks, inhibition of the master kinase will be therapeutically effective, as demonstrated by the spectacular efficacies of BCR-ABL1 inhibitors in CML. If theses kinases function in independent networks or as part of redundant topologies, a combination therapy seems necessary. Therefore, for personalized therapy it will be important to 
map kinase activation profiles onto network connectivity maps that control specific biological responses. This idea is also now adopted by the pharmaceutical industry, especially for kinase inhibitor screening (Box 2).

The idea to correlate phosphotryosine networks with specific biological behaviour was also borne out in studies on the effects of human ERBB2 in human mammary epithelial cells (HMECs) ${ }^{67}$, ${ }^{68}$. ERBB2 is overexpressed in $\sim 25 \%$ of breast cancers and strongly correlates with poor prognosis. Although ERBB2 lacks a known ligand, it can heterodimerise with EGFR and alter EGFR signalling. Quantitative MS based on isobaric tag for relative and absolute quantitation (iTRAQ) labelling was used to map phosphotyrosine networks in parental HMECs and HMECs engineered to overexpress ERBB2 stimulated with either EGF or heregulin (also known as neuregulin 1, NRG1) ${ }^{67}$. The results show that in ERBB2 overexpressing cells EGF stimulates migration through multiple signalling pathways, while heregulin uses a subset of the migration network. Applying regression-based computational modelling identified combinations of phosphorylation sites that correlate with proliferation and migration. Subsequent refinement of the model ${ }^{68}$ permitted the identification of phosphotyrosine network elements that differentially control migration and proliferation. This culminated in the elaboration of nine phosphorylation sites on six proteins, associated with the PI3K pathway and endocytosis, which served as a 'network gauge' that captured the predictive capability of the full model. This result also elegantly demonstrates the analytical power added by computational modelling to the interpretation of complex dynamic datasets. 
Phosphoserine and phosphothreonine antibodies are less well developed than phosphotyrosine antibodies, and most global phosphoproteome studies employ chromatographic enrichment technologies. They usually exploit the propensity of $\mathrm{TiO}_{2}$ or $\mathrm{Fe}^{3+}$ to interact with phosphate groups, and are often combined with a preceding separation step that counterselects phosphopeptides such as strong cation exchange columns ${ }^{69}$. This approach is available in several variations, which enrich overlapping but distinct fractions of the phosphoproteome ${ }^{70}$. It is now possible to identify $10-20,000$ phosphorylation sites. For instance, proteomic analysis of phosphorylation induced by the fms-related tyrosine kinase 3 (FLT3)-internal tandem duplication (ITD) mutant tyrosine kinase quantified $>12,000$ phosphorylation sites and demonstrated subcellular localisation-specific signalling ${ }^{71}$. FLT3-ITD consists of in-frame tandem duplications of the juxtamembrane domain and is the most frequent oncogenic FLT3 mutation in acute myeloid leukaemia (AML), causing constitutive activation of FLT3 and retardation of trafficking through the endoplasmic reticulum (ER). When localized at the ER, FLT3-ITD aberrantly activates STAT5 signalling, but fails to activate the PI3K and ERK pathways that are targets of ligand activated wildtype FLT3. By contrast, when located at the membrane FLT3-ITD preferentially activates PI3K and ERK over STAT5. These pathway specific effects were reflected in the global phosphoproteome and also helped identify putative downstream targets of AKT and proviral integration site (PIM) kinases.

An interesting approach to functional studies is to combine global phosphoproteomics with small interfering RNA (siRNA) knockdown studies. An example is the quantitative proteomic 
assessment of early signalling events in integrin signalling ${ }^{72}$. Integrin mediated cell adhesion and anti-apoptotic signalling is essential for cancer cell spread and invasion. SILAC combined with immobilized metal ion affinity chromatography (IMAC) enrichment showed that >500 phosphorylation sites in 357 proteins changed when integrins interacted with collagen, a common integrin ligand in the extracellular matrix. siRNA screens against 33 of these proteins with kinase or phosphatase activity identified three integrin regulated kinases, p21-activated kinase 2 (PAK2), G protein-coupled receptor kinase 6 (GRK6) and DBF4, which are critically involved in cell migration. Recent evidence suggests that these proteins are deregulated in cancer. PAK2 is hyperphosphorylated in ovarian cancer $^{73}$, while an siRNA screen identified GRK6 as required for the viability of myeloma cells but not normal cells ${ }^{74}$. DBF4 is a regulatory subunit of the cell cycle kinase CDC7 and this complex is overexpressed in a variety of cancer cell lines and cancers ${ }^{75}$.

The focus on phosphoproteomics in recent years, and the application of functional proteomics methods ${ }^{76}$, led to the identification of O-GlcNAcylation as an important PTM in signalling. As O-GlcNAcylation occurs at serine and threonine residues that are also targets for phosphorylation, they mutually exclude each other resulting in a dynamic crosstalk between phosphorylation and O-GlcNAcylation ${ }^{77}$. Although many of the (patho)physiological consequences of this crosstalk remain to be explored it has been implicated in a number of diseases including cancer $^{78}$ and demonstrated to be critical for cellular processes that are important in cancer biology, such as cytokinesis ${ }^{79}$. The application of improved MS techniques (such as electron-transfer 
dissociation) for detecting O-GlcNAcylation ${ }^{80}$ or the parallel detection of phosphorylation and glycosylation ${ }^{81}$ will likely increase attention to this field.

Ubiquitinylation. Ubiquitinylation and the proteosomal pathway play key roles in oncogenesis andcancer ${ }^{82-88}$ (Fig. 3), typified by the tumour suppressor $\mathrm{p} 53^{89,90}$, and are being investigated as pharmaceutical targets ${ }^{91-94}$. Since the early use of functional proteomics to identify the structure and substrate specificity for the S-phase kinase-associated protein 1 (SKP1)-cullin 1 (CUL1)-F-box (SCF) complex ${ }^{95,96}$, this method has continued to play a significant role in the study of ubiquitinylation, in identifying both the components of the ligase complexes and the ubiquitinylation of proteins ${ }^{97}$. Recent studies have also focused on the non-classical ligases, such as the damage-specific DNA binding protein 1 (DDB1)-CUL4A -regulator of cullins 1 (ROC1, also known as RBX1) E3 ubiquitin ligase, a complex that lacks the SKP1-like adaptor and is involved in the regulation of DNA repair ${ }^{98}$. TAP affinity purification of the complex and subsequent MS analysis were used to identify a novel family of $16 \mathrm{WD}$ domain containing proteins that recruit the substrate to the complex ${ }^{99}$. This novel family of proteins was dubbed DDB1-CUL4A-associated WD40 domain proteins (DCAF proteins), and includes DDB2, a protein mutated in the cancer predisposing syndrome xeroderma pigmentosum. However, given the connection to DNA repair a much broader role in cancer and in the sensitivity to DNA damaging treatments seems likely.

Methods are continually being improved for the global identification of ubiquitinylation sites $^{100}$, and to identify substrates in specific pathways ${ }^{101}$. In addition to MS approaches, protein 
arrays are valuable tools for identifying substrates for PTMs. As these assays are done in vitro versatile manipulations and comparisons are possible. Protein microarrays displaying $>8,000$ human proteins were exposed to cell extracts that replicate the mitosis checkpoint and anaphase release in order to identify targets of the anaphase-promoting complex (APC $)^{102}$. APC is a multiprotein complex with E3 ubiquitin ligase activity that promotes cell cycle progression during mitosis. Its substrates include mitotic cyclins, but also many other proteins that orchestrate this cell cycle transition. The array based ubiquitinylation assay compared extracts containing inhibited and activated APC, detecting most of the known substrates, and seven potential new substrates. A similar experiment was used to identify and compare substrates for NEDD4 and NEDD4L ${ }^{103}$. In addition to shared substrates, NEDD4 showed a preference for tyrosine kinases, and NEDD4 knockdown sustained tyrosine kinase signalling. NEDD4 family members regulate a variety of pathways and cellular functions that are implicated in cancer, such as many RTK pathways and transcription factors, and are found overexpressed in breast, prostate and pancreatic cancers ${ }^{104}$.

This brief description of PTM aberrations in cancer is far from complete, and there are many other PTMs that are altered in cancer (Box 3). However, PTMs are emerging as important drug targets. The high hopes and resources invested in kinase inhibitor drugs are inextricably linked to their ability to prevent regulatory phosphorylations of critical substrates. We soon may witness similar prolific efforts to target other PTMs for cancer therapy, such as ubiquitinylation ${ }^{92}$. Notably, the validation of PTM targets is critically enabled by proteomics. PTMs are largely invisible in genomic studies and in order to fully exploit their therapeutic potential it will be necessary to 
understand the dynamics of PTM turnover. It is tempting to speculate that PTM dynamics also contribute to the well known heterogeneity in cancer. Dynamic changes in protein expression and localisation can determine the drug sensitivity of cancer cells ${ }^{105}$ and dynamic changes in PTMs could vastly expand the window of opportunities of cancer cells to escape adverse conditions and therapy.

\section{Towards single cell proteomics}

New techniques enabling the functional analysis of individual cells are increasingly being used to address the heterogeneity of cancer cells, especially in terms of drug resistance. One of the first methods was Phospho-flow, invented by the Nolan group ${ }^{98}$. This technique uses phospho-specific antibodies to label antigens in fixed cells. Subsequent analysis by flow-cytometry gives data on the single cell and population level that allow the investigation of heterogeneity and are applicable for the modelling of cancer signalling pathways ${ }^{106}$. More recently, Cohen et al. tagged $\sim 1000$ individual endogenous proteins in lung cancer cells with a fluorophore and monitored their expression and subcellular location by automated time lapse imaging ${ }^{105}$. Treatment with the topoisomerase 1 inhibitor camptothecin caused the rapid relocation of proteins associated with the mechanism of drug action and slower changes in protein abundance. Interestingly, while most drug induced responses were similar in individual cells across the population, a subset of 24 proteins showed high cell to cell variability a day after drug exposure. The upregulation of two of these proteins, the RNA helicase DEAD box polypeptide 5 (DDX5) and replication factor C1 (RFC1), correlated with drug 
resistance and cell survival, showing that escape mechanisms can result from changes in protein dynamics that are unique to a subset of a cell population. This is an intriguing proposition for the development of drug resistance that warrants further investigation in preclinical cancer models.

A different approach used a microfluidic platform that traps $\sim 1000$ individual cells and analyses their behaviour using imaging, optical indicator dyes and fluorescent antibodies ${ }^{4}$. This platform was used to assess the signalling dynamics of normal human haematopoietic stem cells (HSCs) and CML stem cells (CML-SCs) in response to the second generation BCR-ABL1 inhibitor dasatinib. While significant differences in the responses of individual cells were observed, on the population level dasatinib was more cytotoxic to HSCs than CML-SCs, but strongly and selectively inhibited migration of CML-SCs. Thus, single cell analysis can reveal important information on divergent and synchronised behaviour within cell populations, especially in regard to drug action. It would be fascinating to combine these approaches in order to collect deeper information on cancer cell heterogeneity.

\section{Perspective}

Although the work discussed in this Review represents only a small fraction of studies, it illustrates the power of functional proteomics in mapping cancer signalling pathways. The biggest challenge is to bring the proteomics technologies to clinical applications. Despite impressive progress especially in biomarker discovery [Au; unfortunately the article that discusses this will not be included in the focus issue.], substantial hurdles remain. Most current proteomics technologies are too slow, too 
complex and too expensive to be used in the clinical laboratory, and the existence of many different experimental approaches leaves a deficit in standardisation. However, further innovations that may overcome these barriers are on the horizon. While MS is dominating the field, alternative technologies are appearing. Great progress is to be expected from array-based methods, optical methods and micro-engineering approaches. Although these methods cannot identify proteins de novo, their attractiveness lies in their multiplexing capabilities, throughput and prospect of reducing the costs of analysis. In particular, methods based on flow-cytometry, protein and tissue microarrays and micro-engineered devices are amenable to adaptation to the clinical laboratory.

Our capability to routinely identify or test thousands of proteins per analysis has shifted the bottleneck towards data analysis and interpretation. The increasing use of advanced bioinformatics and systems biology tools is beginning to unlock network properties ${ }^{107}$. Recent examples are the development of a predictor for breast cancer prognosis based on the modularity of protein interaction networks ${ }^{108}$, and the identification of cancer-associated phosphorylation networks through the combined alignment of conserved phosphorylation sites and kinase-substrate networks ${ }^{109}$. This work also showed that we still miss large parts of phosphorylation networks.

As we only begin to apply proteomics to unravel the role of other PTMs, such as ubiquitinylation, sumoylation and glycosylation, in signalling we can expect a further stream of data. It will be a challenging but informative task to understand the regulatory interplay between the different PTMs both on the level of individual proteins and on networks. In addition, these 
investigations may provide molecular functions that can serve as biomarkers for cancer and thereby bridge the current gap between mechanistic understanding and mainly phenomenological markers.

\section{Acknowledgements}

We apologise for omitting many important contributions due to space constraints. We are grateful for funding from Science Foundation Ireland grant No. 06/CE/B1129 (WK) and BBSRC and EPSRC via the RASOR project BB/C511572/1 (AP).

\section{Box 1. Quantitative proteomics.}

Mass spectrometry (MS) is not an a priori quantitative method, but several approaches have been developed to circumvent this problem. Most modern MS approaches are (semi)quantitative, and this has proven key to the success of proteomics to unravel signalling networks. The most used methods comprise ${ }^{110}$ :

2D-polyacrylamide gel electrophoresis (PAGE): The sensitivity, linear dynamic range and robustness of this method has been greatly improved by the use of fluorescent stains and tags ${ }^{111}$, and the analysis significantly simplified by a number of automated software packages. Proteins are identified by MS. 
Isotopic labelling: The ability of MS to resolve heavy and light isotopically labelled molecules has been extensively used to design approaches for quantitation ${ }^{112-115}$. The common labelling methods are: metabolic, typified by stable isotope labelling with amino acids in culture (SILAC) ${ }^{116-118}$. Samples are grown on media containing either a normal or isotopically labelled form of an amino acid or food source ${ }^{119}$. Comparison of protein abundance uses the light and heavy peaks observed in the mass spectrum. SILAC is best suited for cultured cells, but has also been used to label whole $\operatorname{animals}^{120}$. Chemically reactive, isotopically labelled reagents are used to tag the protein or peptides (such as isotope-coded affinity tag, ICAT ${ }^{121}$, isobaric tag for relative and absolute quantitation, iTRAQ $^{122}$ ). For absolute quantification (AQUA, isotopically labelled peptides are added to the sample as an internal standard for quantification ${ }^{123}$.

Label-free: improvements in the robustness and reproducibility of both MS and chromatography paved the way for direct comparisons between MS data sets using retention time, mass and ion intensity $^{124,125}$. As it is uncomplicated and reasonably reliable, it may become the method of choice for proteomics experiments.

Antibody arrays. the repertoire of antibodies with highly selective binding to native or posttranslationally modified protein epitopes has expanded rapidly, and the arrays based on these have been used extensively to quantify the antigen ${ }^{126,127}$. This has now developed into a thriving business, with many companies offering commercial and bespoke custom arrays.

In situ fluorescence: the use of a fusion protein approach, where the protein of interest is genetically fused to green fluorescent protein (GFP) or the range of analogues that have now been developed, 
can determine the cellular localization and abundance of the protein using fluorescence microscopy ${ }^{128}$. Automated methods for tracking cells and determining abundance and localization are only just becoming available.

\section{Box 2. Kinase profiling and kinomics}

Kinase profiling refers to the process of screening kinase inhibitors for activity and selectivity ${ }^{129}$. Given the role that aberrant kinase activity plays in cancer, and the huge efforts in the study of kinases and their substrates and inhibitors, the increase in the number of kinase inhibitors being translated into anti-cancer drugs in recent years is not surprising (it is estimated around a third of all major clinical trials currently involve kinase inhibitors). However, it was only in 2001 when the first kinase inhibitor (imatinib) received clinical approval. 518 kinases were initially predicted from analysis of the human genome $\mathrm{e}^{130}$, of which the vast majority have now been validated, and $>400$ of these are currently available in various screens. However, this is unlikely to be the full story, as new kinases are being identified and tissue specific roles are being elucidated ${ }^{131}$.

Methods to identify kinases generally rely on the use of peptide substrates, either in microtitre plate, bead or array format, that can be used to measure the activity of a set of purified kinases, although with recent improvements ${ }^{132-135}$, methods are getting closer to the native in vivo conditions. Miniaturization through microfluidics and new detection methods are greatly improving sensitivity and reducing costs. The benefits are obvious: it is highly parallelised, so 
many compounds and kinases can be tested, and the quantitative analysis allows the critical question of off-target activity to be addressed.

Kinomics, ${ }^{130}$ is formally the identification of kinases at the genetic, or preferably proteomic, level. However, this should be expanded to include the challenging task of identifying kinase targets and elucidating the cellular pathways and networks in which they are involved and the complex regulation of these. Proteomics approaches coupled to modelling through systems biology methods are in the vanguard of the techniques being applied. A grand challenge remaining is the identification of the actual downstream effector kinases that are the best drug targets. Furthermore, chemical proteomics is a valuable tool to identify on- and off-target interactions of individual kinase inhibitors ${ }^{136}$.

\section{Box 3. Other post-translational modifications (PTMs)}

Protein function is critically regulated by PTMs, and the role of PTM aberrations in the faulty regulation of cancer signalling networks is increasingly appreciated. Proteomics is currently expanding its scope beyond phosphorylation, delivering exciting results on other PTMs such as: Acetylation and methylation: The regulation of gene expression exerted by the acetylation and methylation of lysines and arginines in histones is well established, and the nickname "histone code" highlights the importance of this epigenetic control ${ }^{137}$. Silencing of tumour suppressor gene expression due to subversion of the histone code is well known ${ }^{137}$. However, proteomics has enabled a systematic study of these histone modifications ${ }^{138}$. Importantly, proteomics identified many other 
chromatin-independent targets. For instance, stable isotope labelling with amino acids in culture (SILAC) analysis of breast cancer cells treated with vorinostat, a histone deacetylase (HDAC) inhibitor, revealed changes in the expression of transcription factors, metabolic, structural, chaperone and cell cycle proteins ${ }^{139}$. HDAC6 was identified to inhibit EGFR endocytosis by deacetylating microtubules ${ }^{140}$.

Acylation: A well characterised cancer relevant example is the modification of Ras proteins by farnesylation, geranylation and palmitoylation, which localises Ras proteins to distinct membrane compartments ${ }^{141}$. As Ras mutations are frequent in human cancers, the Ras modifying acylases became major drug targets, unfortunately with disappointing clinical results ${ }^{142}$. Much effort was invested to understand the opportunities and failures, but quantitative proteomic techniques were only recently developed to identify palmitoylated proteins ${ }^{143}$,

Oxidative modifications and cysteine nitrosylation: have also been shown to play an important role in signalling ${ }^{144}$. A role in cancer is highly likely due to the hypoxic and inflammatory environment, but systematic proteomics studies are lagging behind.

\section{References:}

1. Wolf-Yadlin, A., Sevecka, M. \& MacBeath, G. Dissecting protein function and signaling using protein microarrays. Current Opinion in Chemical Biology 13, 398-405 (2009). 
2. Schulz, K.R., Danna, E.A., Krutzik, P.O. \& Nolan, G.P. Single-cell phospho-protein analysis by flow cytometry. Curr Protoc Immunol Chapter 8, Unit 817 (2007).

3. Fournier, F. et al. Biological and biomedical applications of two-dimensional vibrational spectroscopy: proteomics, imaging, and structural analysis. Acc Chem Res 42, 1322-31 (2009).

4. Faley, S.L. et al. Microfluidic single cell arrays to interrogate signalling dynamics of individual, patient-derived hematopoietic stem cells. Lab on a Chip 9, 2659-2664 (2009).

5. Melo, J.V. \& Barnes, D.J. Chronic myeloid leukaemia as a model of disease evolution in human cancer. Nat Rev Cancer 7, 441-53 (2007).

6. Burckstummer, T. et al. An efficient tandem affinity purification procedure for interaction proteomics in mammalian cells. Nat Methods 3, 1013-9 (2006).

7. Gavin, A.C. et al. Proteome survey reveals modularity of the yeast cell machinery. Nature 440, 631-6 (2006).

8. Krogan, N.J. et al. Global landscape of protein complexes in the yeast Saccharomyces cerevisiae. Nature 440, 637-43 (2006).

\section{References 7 and 8 are landmark papers showing the mapping of the yeast interactome by MS}

\section{based proteomics}

9. Bublil, E.M. \& Yarden, Y. The EGF receptor family: spearheading a merger of signaling and therapeutics. Curr Opin Cell Biol 19, 124-34 (2007). 
10. Hammond, D.E. et al. Quantitative Analysis of HGF and EGF-Dependent Phosphotyrosine Signaling Networks. J Proteome Res (2010).

11. Gordus, A. et al. Linear combinations of docking affinities explain quantitative differences in RTK signaling. Molecular Systems Biology 5 (2009).

12. Mitsudomi, T. \& Yatabe, Y. Epidermal growth factor receptor in relation to tumor development: EGFR gene and cancer. FEBS J 277, 301-8 (2010).

13. Dengjel, J., Kratchmarova, I. \& Blagoev, B. Receptor tyrosine kinase signaling: a view from quantitative proteomics. Molecular Biosystems 5, 1112-1121 (2009).

14. von Kriegsheim, A., Preisinger, C. \& Kolch, W. Mapping of Signaling Pathways by Functional Interaction Proteomics. Functional Proteomics: Methods and Protocols, 177-192 (2008).

15. Preisinger, C., von Kriegsheim, A., Matallanas, D. \& Kolch, W. Proteomics and phosphoproteomics for the mapping of cellular signalling networks. Proteomics 8, 4402$4415(2008)$.

16. Ong, S.-E. \& Mann, M. Mass spectrometry-based proteomics turns quantitative. Nat Chem Biol 1, 252-262 (2005).

17. Blagoev, B. \& Mann, M. Quantitative proteomics to study mitogen-activated protein kinases. Methods 40, 243-250 (2006).

18. Cheng, X. Understanding signal transduction through functional proteomics. Expert Review of Proteomics 2, 103-116 (2005). 
19. Andersen, J.S. et al. Proteomic characterization of the human centrosome by protein correlation profiling. Nature 426, 570-4 (2003).

20. Amit, I., Wides, R. \& Yarden, Y. Evolvable signaling networks of receptor tyrosine kinases: relevance of robustness to malignancy and to cancer therapy. Mol Syst Biol 3, 151 (2007).

21. Papanikolaou, N.A. \& Papavassiliou, A.G. Protein complex, gene, and regulatory modules in cancer heterogeneity. Mol Med 14, 543-5 (2008).

22. Luo, J., Solimini, N.L. \& Elledge, S.J. Principles of cancer therapy: oncogene and nononcogene addiction. Cell 136, 823-37 (2009).

23. Henson, E.S. \& Gibson, S.B. in Signal Transduction: Pathways, Mechanisms and Diseases 119-141 (2010).

24. Blagoev, B., Kratchmarova, I., Olsen, J.V. \& Mann, M. in EGFR Signaling Networks in Cancer Therapy 190-198 (2008).

25. Kholodenko, B.N. Cell-signalling dynamics in time and space. Nat Rev Mol Cell Biol 7, 165$176(2006)$.

26. Wiley, H.S., Shvartsman, S.Y. \& Lauffenburger, D.A. Computational modeling of the EGFreceptor system: a paradigm for systems biology. Trends Cell Biol 13, 43-50 (2003).

27. Schulze, W.X., Deng, L. \& Mann, M. Phosphotyrosine interactome of the ErbB-receptor kinase family. Mol Syst Biol 1, 20050008 (2005).

This paper that describes an exhaustive, quantitative MS based proteomics strategy to functionally map protein interactions that mediate ErbB family signalling. 
28. Jones, R.B., Gordus, A., Krall, J.A. \& MacBeath, G. A quantitative protein interaction network for the ErbB receptors using protein microarrays. Nature 439, 168-174 (2006).

Similar to the above this paper describes the use of protein microarrays for the functional mapping of protein interactions in the ErbB family.

29. Alroy, I. \& Yarden, Y. The ErbB signaling network in embryogenesis and oncogenesis: signal diversification through combinatorial ligand-receptor interactions. FEBS Lett 410, 83-6 (1997).

30. Normanno, N. et al. Epidermal growth factor receptor (EGFR) signaling in cancer. Gene 366, 2-16 (2006).

31. von Kriegsheim, A. et al. Cell fate decisions are specified by the dynamic ERK interactome. Nature Cell Biology 11, 1458-U172 (2009).

This paper shows how dynamic changes in ERK interactions with other proteins can specify cell fate decisions in response to different growth factors. An important conclusion is that differentiation is not controlled by a master regulator, but that control is functionally distributed over an entire protein network.

32. Yoon, S. \& Seger, R. The extracellular signal-regulated kinase: Multiple substrates regulate diverse cellular functions. Growth Factors 24, 21-44 (2006).

33. Irvine, D.A., Heaney, N.B. \& Holyoake, T.L. Optimising chronic myeloid leukaemia therapy in the face of resistance to tyrosine kinase inhibitors--a synthesis of clinical and laboratory data. Blood Rev 24, 1-9 (2010). 
34. Nicholson, E. \& Holyoake, T. The chronic myeloid leukemia stem cell. Clin Lymphoma Myeloma 9 Suppl 4, S376-81 (2009).

35. Sawyers, C.L. Signal transduction pathways involved in BCR-ABL transformation. Baillieres Clin Haematol 10, 223-31 (1997).

36. Brehme, M. et al. Charting the molecular network of the drug target Bcr-Abl. Proc Natl Acad Sci U S A 106, 7414-9 (2009).

This paper uses interaction proteomics to map the core protein network involved in signalling by the BCR-ABL1 oncogene, and also examines the effects of the BCR-ABL1 kinase inhibitor imatinib on the network.

37. Brennan, D.J., O’Connor, D.P., Rexhepaj, E., Ponten, F. \& Gallagher, W.M. Antibody-based proteomics: towards the implementation of personalised diagnostic and predictive protocols for cancer patients. Nature Reviews Cancer this issue (2010).

38. Dhillon, A.S., von Kriegsheim, A., Grindlay, J. \& Kolch, W. Phosphatase and feedback regulation of Raf-1 signaling. Cell Cycle 6, 3-7 (2007).

39. Nguyen, A. et al. Kinase suppressor of Ras (KSR) is a scaffold which facilitates mitogenactivated protein kinase activation in vivo. Mol Cell Biol 22, 3035-45 (2002).

40. Kortum, R.L. \& Lewis, R.E. The molecular scaffold KSR1 regulates the proliferative and oncogenic potential of cells. Mol Cell Biol 24, 4407-16 (2004).

41. Lozano, J. et al. Deficiency of kinase suppressor of Ras1 prevents oncogenic ras signaling in mice. Cancer Res 63, 4232-8 (2003). 
42. McKay, M.M., Ritt, D.A. \& Morrison, D.K. Signaling dynamics of the KSR1 scaffold complex. Proceedings of the National Academy of Sciences 106, 11022-11027 (2009).

43. Rajakulendran, T., Sahmi, M., Lefrancois, M., Sicheri, F. \& Therrien, M. A dimerizationdependent mechanism drives RAF catalytic activation. Nature 461, 542-5 (2009).

44. Casar, B., Pinto, A. \& Crespo, P. Essential role of ERK dimers in the activation of cytoplasmic but not nuclear substrates by ERK-scaffold complexes. Mol Cell 31, 708-21 (2008).

45. Liu, L. et al. Proteomic characterization of the dynamic KSR-2 interactome, a signaling scaffold complex in MAPK pathway. Biochimica et Biophysica Acta (BBA) - Proteins \& Proteomics 1794, 1485-1495 (2009).

46. Rauch, J. et al. Heterogeneous nuclear ribonucleoprotein H blocks MST2-mediated apoptosis in cancer cells by regulating A-Raf transcription. Cancer Res 70, 1679-88 (2010).

47. Levchenko, A., Bruck, J. \& Sternberg, P.W. Scaffold proteins may biphasically affect the levels of mitogen-activated protein kinase signaling and reduce its threshold properties. Proc Natl Acad Sci U S A 97, 5818-23 (2000).

48. Dougherty, M.K. et al. KSR2 is a calcineurin substrate that promotes ERK cascade activation in response to calcium signals. Mol Cell 34, 652-62 (2009).

A proteomic comparison of binding partners of KSR1 and KSR2 revealed the selective regulation of KSR2 by the associated calcium regulated phosphatase calcineurin, thus 
demonstrating the regulation of the function of a scaffold protein by a second messenger pathway.

49. Costanzo-Garvey, D.L. et al. KSR2 is an essential regulator of AMP kinase, energy expenditure, and insulin sensitivity. Cell Metab 10, 366-78 (2009).

50. Mazurek, S., Drexler, H.C., Troppmair, J., Eigenbrodt, E. \& Rapp, U.R. Regulation of pyruvate kinase type M2 by A-Raf: a possible glycolytic stop or go mechanism. Anticancer Res 27, 3963-71 (2007).

51. Christofk, H.R. et al. The M2 splice isoform of pyruvate kinase is important for cancer metabolism and tumour growth. Nature 452, 230-3 (2008).

52. Macek, B., Mann, M. \& Olsen, J.V. Global and Site-Specific Quantitative Phosphoproteomics: Principles and Applications. Annual Review of Pharmacology and Toxicology 49, 199-221 (2009).

53. Oppermann, F.S. et al. Large-scale proteomics analysis of the human kinome. Mol Cell Proteomics 8, 1751-64 (2009).

54. Mayya, V. \& Han, D.K. Phosphoproteomics by mass spectrometry: insights, implications, applications and limitations. Expert Review of Proteomics 6, 605-618 (2009).

55. Olsen, J.V. et al. Global, in vivo, and site-specific phosphorylation dynamics in signaling networks. Cell 127, 635-648 (2006).

This paper reports the large scale quantitative mapping of phosphorylation events triggered by EGF revealing new insights into the role of phosphorylation in signalling. 
56. Schmezle, K. \& White, F.M. Phosphoproteomic approaches to elucidate cellular signaling networks. Current Opinion in Biotechnology 17, 406-414 (2006).

57. Blagoev, B., Ong, S.E., Kratchmarova, I. \& Mann, M. Temporal analysis of phosphotyrosinedependent signaling networks by quantitative proteomics. Nat Biotechnol 22, 1139-45 (2004).

58. Kratchmarova, I., Blagoev, B., Haack-Sorensen, M., Kassem, M. \& Mann, M. Mechanism of divergent growth factor effects in mesenchymal stem cell differentiation. Science 308, 1472-7 (2005).

59. Ball, S.G., Shuttleworth, C.A. \& Kielty, C.M. Platelet-derived growth factor receptors regulate mesenchymal stem cell fate: implications for neovascularization. Expert Opin Biol Ther 10, 57-71 (2010).

60. Heibeck, T.H. et al. An Extensive Survey of Tyrosine Phosphorylation Revealing New Sites in Human Mammary Epithelial Cells. Journal of Proteome Research 8, 3852-3861 (2009).

61. Gan, H.K., Kaye, A.H. \& Luwor, R.B. The EGFRvIII variant in glioblastoma multiforme. J Clin Neurosci 16, 748-54 (2009).

62. Huang, P.H. et al. Quantitative analysis of EGFRvIII cellular signaling networks reveals a combinatorial therapeutic strategy for glioblastoma. Proc Natl Acad Sci U S A 104, 12867-72 (2007).

63. Guo, A. et al. Signaling networks assembled by oncogenic EGFR and c-Met. Proc Natl Acad Sci U S A 105, 692-7 (2008). 
64. Amit, I. et al. A module of negative feedback regulators defines growth factor signaling. Nat Genet 39, 503-12 (2007).

65. Govindan, R. A review of epidermal growth factor receptor/HER2 inhibitors in the treatment of patients with non-small-cell lung cancer. Clin Lung Cancer 11, 8-12 (2010).

66. Rikova, K. et al. Global survey of phosphotyrosine signaling identifies oncogenic kinases in lung cancer. Cell 131, 1190-203 (2007).

Proteomic profiling of tyrosine phosphorylation in a set of approximately 200 lung cancers identified characteristic phosphorylation signatures and known as well as new tyrosine kinases involved in lung cancer.

67. Wolf-Yadlin, A. et al. Effects of HER2 overexpression on cell signaling networks governing proliferation and migration. Mol Syst Biol 2, 54 (2006).

68. Kumar, N., Wolf-Yadlin, A., White, F.M. \& Lauffenburger, D.A. Modeling HER2 effects on cell behavior from mass spectrometry phosphotyrosine data. PLoS Comput Biol 3, e4 (2007).

69. Villen, J. \& Gygi, S.P. The SCX/IMAC enrichment approach for global phosphorylation analysis by mass spectrometry. Nat Protoc 3, 1630-8 (2008).

70. Bodenmiller, B., Mueller, L.N., Mueller, M., Domon, B. \& Aebersold, R. Reproducible isolation of distinct, overlapping segments of the phosphoproteome. Nat Methods 4, 231-7 (2007).

71. Choudhary, C. et al. Mislocalized activation of oncogenic RTKs switches downstream signaling outcomes. Mol Cell 36, 326-39 (2009). 
72. Chen, Y. et al. Combined Integrin Phosphoproteomic Analyses and Small Interfering RNABased Functional Screening Identify Key Regulators for Cancer Cell Adhesion and Migration. Cancer Res 69, 3713-3720 (2009).

This study reports the identification of proteins that mediate integrin regulation of cell adhesion and migration using a combination of quantitative proteomics and functional siRNA experiments.

73. Siu, M.K. et al. Differential expression and phosphorylation of Pak1 and Pak2 in ovarian cancer: effects on prognosis and cell invasion. Int J Cancer 127, 21-31 (2010).

74. Tiedemann, R.E. et al. Kinome-wide RNAi studies in human multiple myeloma identify vulnerable kinase targets, including a lymphoid-restricted kinase, GRK6. Blood 115, 1594$604(2010)$.

75. Bonte, D. et al. Cdc7-Dbf4 kinase overexpression in multiple cancers and tumor cell lines is correlated with p53 inactivation. Neoplasia 10, 920-31 (2008).

76. Hall, M.C. Proteomics Modifies Our Understanding of Cell Cycle Complexity. Sci. Signal. 3, pe4- (2010).

77. Shimoji, S., Park, K. \& Hart, G.W. Dynamic Crosstalk between GlcNAcylation and Phosphorylation: Roles in Signaling, Transcription and Human Disease (Supplementary Material). Current Signal Transduction Therapy 5, 25-40 (2010).

78. Hart, G.W., Housley, M.P. \& Slawson, C. Cycling of O-linked [beta]-N-acetylglucosamine on nucleocytoplasmic proteins. Nature 446, 1017-1022 (2007). 
79. Wang, Z. et al. Extensive Crosstalk Between O-GlcNAcylation and Phosphorylation Regulates Cytokinesis. Sci. Signal. 3, ra2- (2010).

\section{This paper shows that $\mathrm{O}$-GlcNAcylation competes with phosphorylation and plays a major role} in the regulation of mitotic spindle assembly and cytokinesis.

80. Chalkley, R.J., Thalhammer, A., Schoepfer, R. \& Burlingame, A.L. Identification of protein O-GlcNAcylation sites using electron transfer dissociation mass spectrometry on native peptides. Proceedings of the National Academy of Sciences 106, 8894-8899 (2009).

81. Wu, S.L. et al. Dynamic profiling of the post-translational modifications and interaction partners of epidermal growth factor receptor signaling after stimulation by epidermal growth factor using Extended Range Proteomic Analysis (ERPA). Mol Cell Proteomics 5, 1610-27 (2006).

82. Yang, W.L., Wu, C.Y., Wu, J. \& Lin, H.K. Regulation of Akt signaling activation by ubiquitination. Cell Cycle 9, 486-497 (2010).

83. Kitagawa, K., Kotake, Y. \& Kitagawa, M. Ubiquitin-mediated control of oncogene and tumor suppressor gene products. Cancer Science 100, 1374-1381 (2009).

84. Ardley, H.C. Ring Finger Ubiquitin Protein Ligases and Their Implication to the Pathogenesis of Human Diseases. Current Pharmaceutical Design 15, 3697-3715 (2009).

85. Vlachostergios, P.J., Patrikidou, A., Daliani, D.D. \& Papandreou, C.N. The ubiquitinproteasome system in cancer, a major player in DNA repair. Part 1: post-translational regulation. Journal of Cellular and Molecular Medicine 13, 3006-3018 (2009). 
86. Vlachostergios, P.J., Patrikidou, A., Daliani, D.D. \& Papandreou, C.N. The ubiquitinproteasome system in cancer, a major player in DNA repair. Part 2: transcriptional regulation. J Cell Mol Med 13, 3019-31 (2009).

87. Nakayama, K.I. \& Nakayama, K. Ubiquitin ligases: cell-cycle control and cancer. Nature Reviews Cancer 6, 369-381 (2006).

88. Mani, A. \& Gelmann, E.P. The Ubiquitin-Proteasome Pathway and Its Role in Cancer. J Clin Oncol 23, 4776-4789 (2005).

89. Coutts, A.S., Adams, C.J. \& La Thangue, N.B. p53 ubiquitination by Mdm2: A never ending tail? DNA Repair 8, 483-490 (2009).

90. Marine, J.C. \& Lozano, G. Mdm2-mediated ubiquitylation: p53 and beyond. Cell Death and Differentiation 17, 93-102 (2010).

91. Yang, Y.L., Kitagaki, J., Wang, H., Hou, D.X. \& Perantoni, A.O. Targeting the ubiquitinproteasome system for cancer therapy. Cancer Science 100, 24-28 (2009).

92. Hoeller, D. \& Dikic, I. Targeting the ubiquitin system in cancer therapy. Nature 458, 438444 (2009).

93. Ande, S.R., Chen, J.J. \& Maddika, S. The ubiquitin pathway: An emerging drug target in cancer therapy. European Journal of Pharmacology 625, 199-205 (2009).

94. Nalepa, G., Rolfe, M. \& Harper, J.W. Drug discovery in the ubiquitin-proteasome system. Nature Reviews Drug Discovery 5, 596-613 (2006).

95. Tang, X. et al. in Methods in Enzymology 433-458 (Academic Press, 2005). 
96. Bai, C. et al. SKP1 Connects Cell Cycle Regulators to the Ubiquitin Proteolysis Machinery through a Novel Motif, the F-Box. Cell 86, 263-274 (1996).

97. Radivojac, P. et al. Identification, analysis, and prediction of protein ubiquitination sites. Proteins-Structure Function and Bioinformatics 78, 365-380 (2010).

98. Irish, J.M. et al. Single cell profiling of potentiated phospho-protein networks in cancer cells. Cell 118, 217-28 (2004).

99. Angers, S. et al. Molecular architecture and assembly of the DDB1-CUL4A ubiquitin ligase machinery. Nature 443, 590-593 (2006).

100. Jeram, S.M. et al. An improved SUMmOn-based methodology for the identification of ubiquitin and ubiquitin-like protein conjugation sites identifies novel ubiquitin-like protein chain linkages. Proteomics 10, 254-265 (2010).

101. Burande, C.F. et al. A Label-free Quantitative Proteomics Strategy to Identify E3 Ubiquitin Ligase Substrates Targeted to Proteasome Degradation. Molecular \& Cellular Proteomics 8, 1719-1727 (2009).

102. Merbl, Y. \& Kirschner, M.W. Large-scale detection of ubiquitination substrates using cell extracts and protein microarrays. Proceedings of the National Academy of Sciences of the United States of America 106, 2543-2548 (2009).

103. Persaud, A. et al. Comparison of substrate specificity of the ubiquitin ligases Nedd4 and Nedd4-2 using proteome arrays. Molecular Systems Biology 5, Article No.: 333 (2009). 
The above two papers demonstrate the use of protein microarrays to systematically identify substrates for ubiquitin ligases on a large scale.

104. Chen, C. \& Matesic, L.E. The Nedd4-like family of E3 ubiquitin ligases and cancer. Cancer Metastasis Rev 26, 587-604 (2007).

105. Cohen, A.A. et al. Dynamic Proteomics of Individual Cancer Cells in Response to a Drug. Science 322, 1511-1516 (2008).

106. Irish, J.M., Kotecha, N. \& Nolan, G.P. Mapping normal and cancer cell signalling networks: towards single-cell proteomics. Nat Rev Cancer 6, 146-55 (2006).

107. Kreeger, P.K. \& Lauffenburger, D.A. Cancer systems biology: a network modeling perspective. Carcinogenesis 31, 2-8 (2010).

108. Taylor, I.W. et al. Dynamic modularity in protein interaction networks predicts breast cancer outcome. Nat Biotechnol 27, 199-204 (2009).

This paper shows that dynamic changes in the organisation of cellular protein-protein interactions differ between breast cancer patients and can be used to predict prognosis.

109. Tan, C.S. et al. Comparative analysis reveals conserved protein phosphorylation networks implicated in multiple diseases. Sci Signal 2, ra39 (2009).

Here, the reconstruction of kinase-substrate networks based on phosphoproteomics data and evolutionary conservation of the phoshorylation sites revealed that several diseases including cancer affect conserved parts of the phosphorylation networks. 
110. Fenselau, C. A review of quantitative methods for proteomic studies. Journal of Chromatography B 855, 14-20 (2007).

111. Van den Bergh, G. \& Arckens, L. Recent advances in 2D electrophoresis: an array of possibilities. Expert Review of Proteomics 2, 243-252 (2005).

112. Pan, S. \& Aebersold, R. Quantitative proteomics by stable isotope labeling and mass spectrometry. Methods Mol Biol 367, 209-18 (2007).

113. Wepf, A., Glatter, T., Schmidt, A., Aebersold, R. \& Gstaiger, M. Quantitative interaction proteomics using mass spectrometry. Nat Methods 6, 203-5 (2009).

114. Linscheid, M.W., Ahrends, R., Pieper, S. \& Kuhn, A. Liquid chromatography-mass spectrometry-based quantitative proteomics. Methods Mol Biol 564, 189-205 (2009).

115. Bantscheff, M., Schirle, M., Sweetman, G., Rick, J. \& Kuster, B. Quantitative mass spectrometry in proteomics: a critical review. Analytical and Bioanalytical Chemistry 389, 1017-1031 (2007).

116. Everley, P.A., Krijgsveld, J., Zetter, B.R. \& Gygi, S.P. Quantitative cancer proteomics: stable isotope labeling with amino acids in cell culture (SILAC) as a tool for prostate cancer research. Mol Cell Proteomics 3, 729-35 (2004).

117. Mann, M. Functional and quantitative proteomics using SILAC. Nat Rev Mol Cell Biol 7, 952-8 (2006).

118. Ong, S.E. et al. Stable isotope labeling by amino acids in cell culture, SILAC, as a simple and accurate approach to expression proteomics. Mol Cell Proteomics 1, 376-86 (2002). 
119. Beynon, R.J. \& Pratt, J.M. Metabolic Labeling of Proteins for Proteomics. Molecular \& Cellular Proteomics 4, 857-872 (2005).

120. Kruger, M. et al. SILAC mouse for quantitative proteomics uncovers kindlin-3 as an essential factor for red blood cell function. Cell 134, 353-64 (2008).

121. Gygi, S.P. et al. Quantitative analysis of complex protein mixtures using isotope-coded affinity tags. Nat Biotech 17, 994-999 (1999).

122. Ross, P.L. et al. Multiplexed Protein Quantitation in Saccharomyces cerevisiae Using Amine-reactive Isobaric Tagging Reagents. Molecular \& Cellular Proteomics 3, 1154-1169 (2004).

123. Gerber, S.A., Rush, J., Stemman, O., Kirschner, M.W. \& Gygi, S.P. Absolute quantification of proteins and phosphoproteins from cell lysates by tandem MS. Proceedings of the National Academy of Sciences of the United States of America 100, 6940-6945 (2003).

124. Haqqani, A.S., Kelly, J.F. \& Stanimirovic, D.B. Quantitative protein profiling by mass spectrometry using label-free proteomics. Methods Mol Biol 439, 241-56 (2008).

125. Negishi, A. et al. Large-scale quantitative clinical proteomics by label-free liquid chromatography and mass spectrometry. Cancer Sci 100, 514-9 (2009).

126. Kopf, E. \& Zharhary, D. Antibody arrays--An emerging tool in cancer proteomics. The International Journal of Biochemistry \& Cell Biology 39, 1305-1317 (2010). 
127. Haab, B., Dunham, M. \& Brown, P. Protein microarrays for highly parallel detection and quantitation of specific proteins and antibodies in complex solutions. Genome Biology 1, preprint0001.1 - preprint0001.22(2000).

128. Rino, J., Braga, J., Henriques, R. \& Carmo-Fonseca, M. Frontiers in fluorescence microscopy. International Journal of Developmental Biology 53, 1569-1579 (2009).

129. Goldstein, D.M., Gray, N.S. \& Zarrinkar, P.P. High-throughput kinase profiling as a platform for drug discovery. Nat Rev Drug Discov 7, 391-397 (2008).

130. Manning, G., Whyte, D.B., Martinez, R., Hunter, T. \& Sudarsanam, S. The protein kinase complement of the human genome. Science 298, 1912-34 (2002).

131. Fedorov, O., Muller, S. \& Knapp, S. The (un)targeted cancer kinome. Nat Chem Biol 6, 166169 (2010).

132. Kubota, K. et al. Sensitive multiplexed analysis of kinase activities and activity-based kinase identification. Nat Biotechnol 27, 933-40 (2009).

133. Khan, I.H. et al. Microbead arrays for the analysis of ErbB receptor tyrosine kinase activation and dimerization in breast cancer cells. Assay Drug Dev Technol 8, 27-36 (2010).

134. Wu, D., Sylvester, J.E., Parker, L.L., Zhou, G. \& Kron, S.J. Peptide reporters of kinase activity in whole cell lysates. Biopolymers 94, 475-86 (2010).

135. Parikh, K., Peppelenbosch, M.P. \& Ritsema, T. Kinome profiling using peptide arrays in eukaryotic cells. Methods Mol Biol 527, 269-80, x (2009). 
136. Rix, U. \& Superti-Furga, G. Target profiling of small molecules by chemical proteomics. Nat Chem Biol 5, 616-624 (2009).

137. Chi, P., Allis, C.D. \& Wang, G.G. Covalent histone modifications--miswritten, misinterpreted and mis-erased in human cancers. Nat Rev Cancer 10, 457-69 (2010).

138. Young, N.L. et al. High throughput characterization of combinatorial histone codes. Mol Cell Proteomics 8, 2266-84 (2009).

139. Zhou, Q. et al. Screening for therapeutic targets of vorinostat by SILAC-based proteomic analysis in human breast cancer cells. Proteomics 10, 1029-39 (2010).

140. Deribe, Y.L. et al. Regulation of epidermal growth factor receptor trafficking by lysine deacetylase HDAC6. Sci Signal 2, ra84 (2009).

141. Rocks, O., Peyker, A. \& Bastiaens, P.I. Spatio-temporal segregation of Ras signals: one ship, three anchors, many harbors. Curr Opin Cell Biol 18, 351-7 (2006).

142. Hao, D. \& Rowinsky, E.K. Inhibiting signal transduction: recent advances in the development of receptor tyrosine kinase and Ras inhibitors. Cancer Invest 20, 387-404 (2002).

143. Yang, W., Di Vizio, D., Kirchner, M., Steen, H. \& Freeman, M.R. Proteome scale characterization of human S-acylated proteins in lipid raft-enriched and non-raft membranes. Mol Cell Proteomics 9, 54-70 (2010). 
144. Spickett, C.M., Pitt, A.R., Morrice, N. \& Kolch, W. Proteomic analysis of phosphorylation, oxidation and nitrosylation in signal transduction. Biochim Biophys Acta 1764, 1823-41 (2006).

145. Wilson, K.J., Gilmore, J.L., Foley, J., Lemmon, M.A. \& Riese, D.J., 2nd. Functional selectivity of EGF family peptide growth factors: implications for cancer. Pharmacol Ther 122, 1-8 (2009).

146. Citri, A. \& Yarden, Y. EGF-ERBB signalling: towards the systems level. Nat Rev Mol Cell Biol 7, 505-16 (2006).

147. Yao, Z. \& Seger, R. The ERK signaling cascade--views from different subcellular compartments. Biofactors 35, 407-16 (2009).

148. McKay, M.M. \& Morrison, D.K. Integrating signals from RTKs to ERK/MAPK. Oncogene 26, 3113-21 (2007).

149. Dhillon, A.S., Hagan, S., Rath, O. \& Kolch, W. MAP kinase signalling pathways in cancer. Oncogene 26, 3279-90 (2007).

150. Preisinger, C. \& Kolch, W. The Bcr-Abl kinase regulates the actin cytoskeleton via a GADS/Slp-76/Nck1 adaptor protein pathway. Cell Signal 22, 848-56 (2010).

151. Dyson, J.M. et al. The SH2-containing inositol polyphosphate 5-phosphatase, SHIP-2, binds filamin and regulates submembraneous actin. J Cell Biol 155, 1065-79 (2001).

152. Ikeda, F. \& Dikic, I. Atypical ubiquitin chains: new molecular signals. 'Protein Modifications: Beyond the Usual Suspects' review series. EMBO Rep 9, 536-42 (2008). 
153. Schwartz, A.L. \& Ciechanover, A. Targeting proteins for destruction by the ubiquitin system: implications for human pathobiology. Annu Rev Pharmacol Toxicol 49, 73-96 (2009).

\section{Figure Legends}

Fig. 1. The EGFR signalling network. Upon activation (by ligand binding), the epidermal growth factor receptor (EGFR, also known as ERBB1) autophosphorylates tyrosine residues in its cytoplasmic domain, which serve as docking sites for the assembly of protein complexes that transduce EGF signals to generate specific biological responses. It is unlikely that the full complement of binding partners is recruited to each receptor, and rather more plausible that individual EGFRs form complexes with different compositions. In addition, all four members of the ErbB family (EGFR, ERBB2 (also known as HER2), ERBB3 and ERBB4) can form heterodimers (not shown), which share common binders but due to the different representation of docking sites assemble receptor complexes with different signalling properties ${ }^{27}$. The EGFR network has been reviewed in detail elsewhere ${ }^{145,146}$, and only the better characterised downstream pathways are shown here. Kinases are light blue; scaffolds are dark blue; adaptor proteins are yellow; G-proteins are green; transcription factors are orange; phospholipase $\mathrm{C} \gamma(\mathrm{PLC} \gamma)$ is a phospholipase; CBL is a ubiquitin ligase; son of sevenless homologue (SOS) is a guanine nucleotide exchange factor. Small molecule second messengers are in red: phosphatidylinositol 4,5-bisphosphate (PIP2),; diacylglycerol (DAG), inositol 1,4,5-triphosphate (IP3), and $\mathrm{Ca}^{2+}$. The pathway discussed in this 
review is the ERK pathway, which is initiated through the recruitment of a protein complex containing the Src homology 2 domain containing (SHC) and growth factor receptor-bound protein 2 (GRB2) adaptor proteins and the exchange factor SOS to the activated EGFR. SOS exchanges GDP for GTP on Ras, which induces a conformational change that enables Ras to bind Raf kinases with high affinity. Raf activation is a complex process involving dephosphorylation, phosphorylation, homo- and hetero-dimerisation and binding to the kinase suppressor of ras 1 (KSR1) scaffold ${ }^{147-149}$. Activated Raf phosphorylates and activates MEK, which in turn phosphorylates and activates ERK. ERK exerts its different biological effects through a large number of substrates $^{32}$ including transcription factors. AP1, activator protein 1; CAMK, calcium/calmodulin-dependent protein kinase; CREB, cAMP responsive element binding protein; EGR1, early growth response 1; FAK, focal adhesion kinase; MEF2, myocyte enhancer factor 2; PKC, protein kinase C; STAT, signal transducer and activator of transcription. Please check definitions carefully.]

Fig. 2. The BCR-ABL1 signalling network. Breakpoint cluster region (BCR)-ABL1 activates downstream signalling pathways through a core network of 7 closely associated proteins ${ }^{36}$. They comprise adaptor proteins (blue), phosphatases (orange), and the regulatory p85 subunit of PI3K (green) [Au; please note that this was changed according to the redrawn figures, which I will send to you soon.]. Selected downstream signalling pathways are depicted in light green and biological outcomes in yellow. Most biological effects are regulated through multiple pathways, for example 
actin polymerisation is regulated through the GRB2-related adapter protein 2 (GRAP2, also known as GADS)- lymphocyte cytosolic protein 2 (LCP2, also known as SLP76)-Nck adaptor pathway ${ }^{150}$, or SHIP2 regulation of phosphatidylinositol-3,4,5-trisphosphate levels ${ }^{151}$. The scheme is highly simplified for clarity. Arrows do not imply direct connections and may summarize several steps. Based on data from Brehme et al. ${ }^{36}$ AP2, adaptor protein complex 2; CSK, c-src tyrosine kinase; GRB2, growth factor receptor-bound protein 2; SHC1, Src homology 2 domain containing 1; STS1, suppressor of T-cell receptor signaling 1.

Fig. 3. The ubiquitin system. Ubiquitin (Ub) is a small protein that is transferred as a versatile regulatory post-translational modification (PTM) to target proteins in a three step process ${ }^{152}$. In the first step an E1 ubiquitin-activating enzyme uses ATP to form a covalent thioester with ubiquitin. E1 then transfers the ubiquitin moiety onto an ubiquitin-conjugating enzyme E2. E2 associates with one of several hundred E3 ubiquitin ligases which provide specificity by also binding the target protein. The E2 binding and target recognition domains can reside in one protein (as shown) or in different proteins that a part of a ligase complex. Finally, the ubiquitin moiety is transferred to a lysine residue in the target protein directly or through an E3-ubiquitin ligase. Further ubiquitins can be added to lysines in ubiquitin itself. Dependent on the number of ubiquitin moieties transferred and the linkage type ubiquitinylation can have different functions that are often derailed in cancer ${ }^{83}$ and many other diseases ${ }^{153}$. 


\section{At-a-glance summary}

- Signalling pathways are commonly deranged in cancer and quantitative proteomics offers powerful approaches to map these pathways and their aberrations in cancer.

- Hubs in signalling pathways feature multiple protein interactions, which are involved in information processing and specification of the biological responses. These networks can be mapped by interaction proteomics to reveal molecular mechanisms of transformation as well as potential targets for therapeutic interventions.

- The oncogenic actions of the epidermal growth factor receptor (EGFR) network and the breakpoint cluster region (BCR)-ABL1 oncogene rely on the dynamic assembly of multiprotein complexes, which activate multiple downstream pathways that cooperate in transformation. In EGFR networks the oncogenic potential increases with the number of downstream pathways being activated.

- The dynamic assembly of protein complexes is regulated by post-translational modifications (PTMs), such as phosphorylation. Advances in phosphoproteomics allow both the targeted and global mapping of phosphorylation networks confirming that kinase networks play major roles in cancer and offer numerous new targets for therapeutic intervention.

- In addition to phosphorylation, a role for PTMs in the regulation of cancer cell biology is becoming increasingly recognised. For instance, proteomic studies of ubiquitination are beginning to unravel extensive alterations that contribute to key alterations of cancer, such as growth factor receptor activation, transcription factor function, protein localisation and degradation. 
- Dynamic changes in protein abundance and PTMs may also contribute to cancer cell heterogeneity, and new proteomics technologies based on optical, spectroscopic and microarray methods are being developed to analyse individual cells.

\section{Glossary}

Chemical biology. Using chemicals, usually drugs or drug-like compounds, to probe biological systems to measure the response of biological systems to perturbations, but in proteomics it also increasingly refers to the use of affinity reagents to enrich classes of proteins for further analysis.

Chemical genetics. A part of chemical biology that focuses on the use of chemicals to explore genetic systems as well as genetic factors that determine drug sensitivity.

Matrix management. A flexible management approach that assigns people with the required skill sets to projects, typically drawing expertise from different departments. This comparison is used to illustrate that a protein with a defined molecular function, such as a kinase, can be employed by several different pathways.

Modularization. The grouping of different functions into a single unit (module) so that the output of the module can be treated as a single functional entity, such as the ability of different combinations of components of protein complexes to achieve the same output.

SH2 domain. Src homology 2 domains were first discovered as conserved domain in the Src kinase family. SH2 domains recognize short peptide motifs containing a phosphorylated tyrosine residue., and thus function as phosphotyrosine dependent protein interaction sites. 
Non-oncogene addiction. This occurs when the action of oncogenes needs to be supported by apparently normally functioning signalling pathways that allow the mutated oncogene to develop its transforming activity.

Stable isotope labeling with amino acids in cell culture (SILAC). This method involves the in vivo metabolic labelling of samples with amino acids that carry stable (nonradioactive) heavy isotope substitutions of atoms which when analyzed by MS produces so-called 'conjugated' peptide peaks, which originate from the same protein but show a characteristic mass shift corresponding to the mass difference between the light and heavy label; the relative intensity of conjugated peak pairs provides the relative abundance in the two samples.

Nodes. Describes objects in graph, while the connections between objects are termed edges, and in signalling networks nodes represent proteins (or genes, if they are based on genetic information), and edges represent the relationship between the nodes, such as binding, regulation or modification.

Scaffold proteins. Proteins that are able to simultaneously bind two or more other proteins, and thereby facilitate physical and functional interactions between their client proteins.

The Warburg effect. Named after a discovery made by the German biochemist Otto Warburg in the 1920's that cancer cells predominantly use anaerobic glycolysis rather than oxidative phosphorylation even when oxygen is abundant, and as a result pyruvate is converted to lactate instead of being oxidised by the mitochondria. 
O-linked N-acetylglucosamine acylation. (O-GlcNAcylation) A form of glycosylation found in nuclear and cytosolic proteins where O-GlcNAc is added to the hydroxyl groups of serine and threonine residues that can also serve as phosphorylation sites.

iTRAQ (isobaric tag for relative and absolute quantitation). A stable isotope labelling method for the quantitation of peptides by MS, where a molecule containing either normal or heavy isotopes is used to chemically modify the proteins or peptides from each individual sample, and the fragmentation of the labelled molecules gives rise to specific reporter ions that can be used to measure the relative amounts present in each sample.

IMAC (immobilized metal ion affinity chromatography).A method for the enrichment of phosphopeptides exploiting the propensity of metal ions like iron or gallium to bind phosphate groups.

Electron transfer dissociation (ETD).A recently introduced MS method for the fragmentation of molecules by transferring electrons from anion radicals to the positively charged ions; it is a non-ergodic process (rapid, kinetically controlled) so energy is not redistributed and many bonds are broken in the molecule, not just the weakest ones as seen in collision-induced dissociation.

Collision-induced dissociation (CID).A common MS fragmentation method where energy is transferred to ions thermally through collisions with an inert gas, giving a vibrational activation fragmentation process that is thermodynamic (the energy has time to redistribute through the molecule) and tends to break mainly the weakest bonds in the structure. 
Cyclins. Regulatory subunits that are essential for the activity of cell cycle dependent kinases (CDKs). Their name derives from their periodic expression during the cell cycle, which is due to the regulated degradation by the ubiquitin-proteasome system that is thought to drive the cell cycle.

WD40 domains. Protein domain consisting of 4-16 repeats of an approximately 40 amino acid long motif ending with a W-D (tryptophan-aspartic acid) di-peptide. The WD40 domains form a circular $\beta$-sheet propeller structure that serves as a structural platform for protein interactions, while the specificity of the interactions is determined by sequences outside of the WD repeats.

Micro-engineering. The use of micro-fabricated devices with small (micron) scale features (channels, wells, vessels, etc) to allow the processing of small volumes of fluid.

Micro-Western Arrays.A miniaturized version of the traditional Western blotting procedure, where robotics are used to mutiplex the running and blotting of miniaturized gels.

\section{Authors' Biographies}

Andrew Pitt is a Reader in the Integrative and Systems Biology section of the Faculty of Biomedical and Life Sciences at the University of Glasgow. He received his D.Phil in bioorganic chemistry from the University of Oxford, before moving to Glasgow via a Fellowship at the University of Cambridge and a Lectureship at the 
University of Strathclyde. His research focuses on the development of new technologies and methodologies for post-genomic research, especially proteomics and metabolomics, chemical biology and data generation and analysis for systems biology.

Walter Kolch obtained a MD from the University of Vienna, Austria. He worked in academia, research institutions and pharmaceutical industry. Currently, he is Director of Systems Biology Ireland and the Conway Institute of Biomolecular \& Biomedical Research at University College Dublin, Ireland. His research interests developed from the study of oncogenes to include signal transduction, proteomics and systems biology with a view to understand how biochemical signalling networks specify biological behaviour. 
Fig. 1

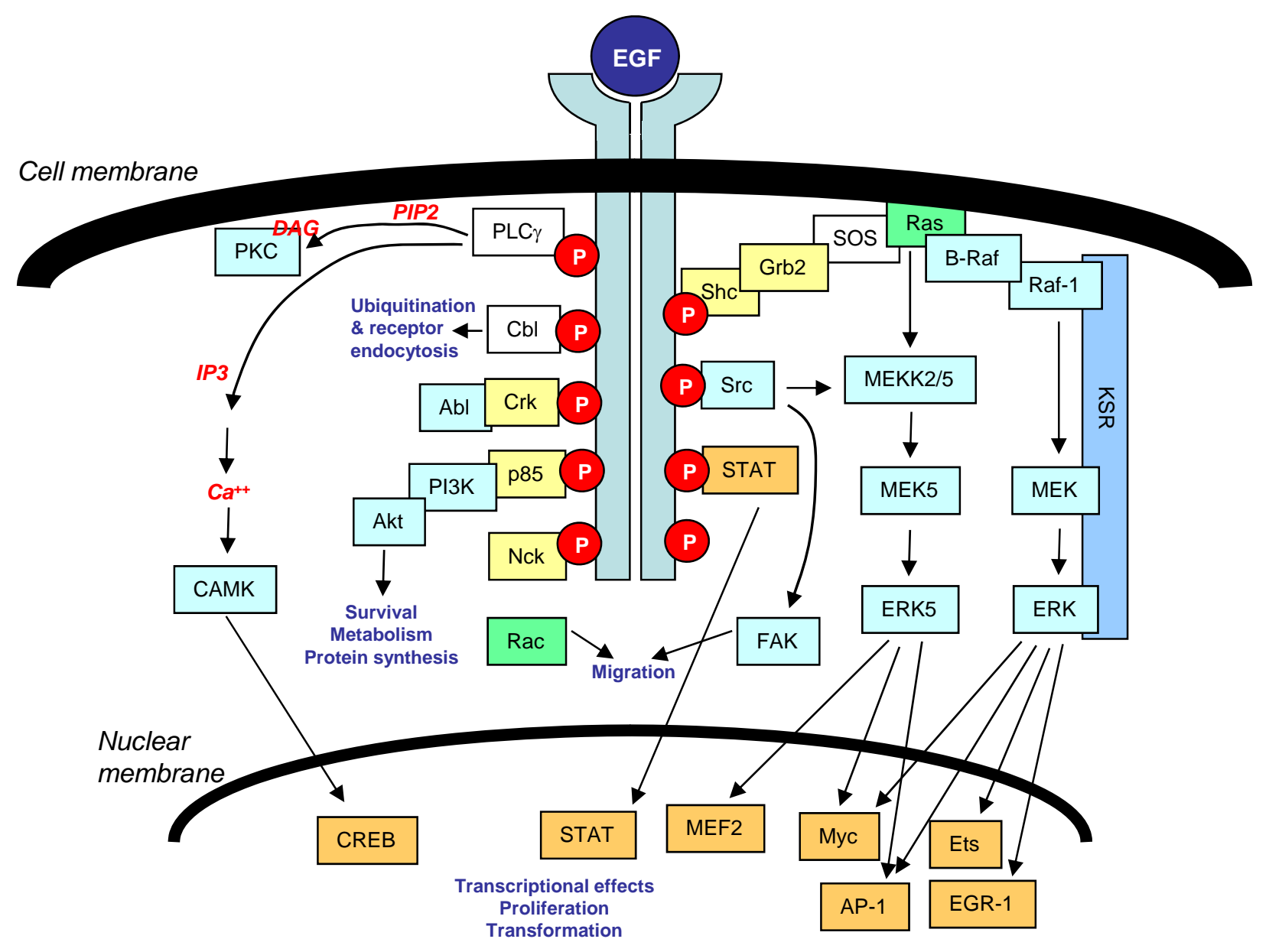


Fig. 2

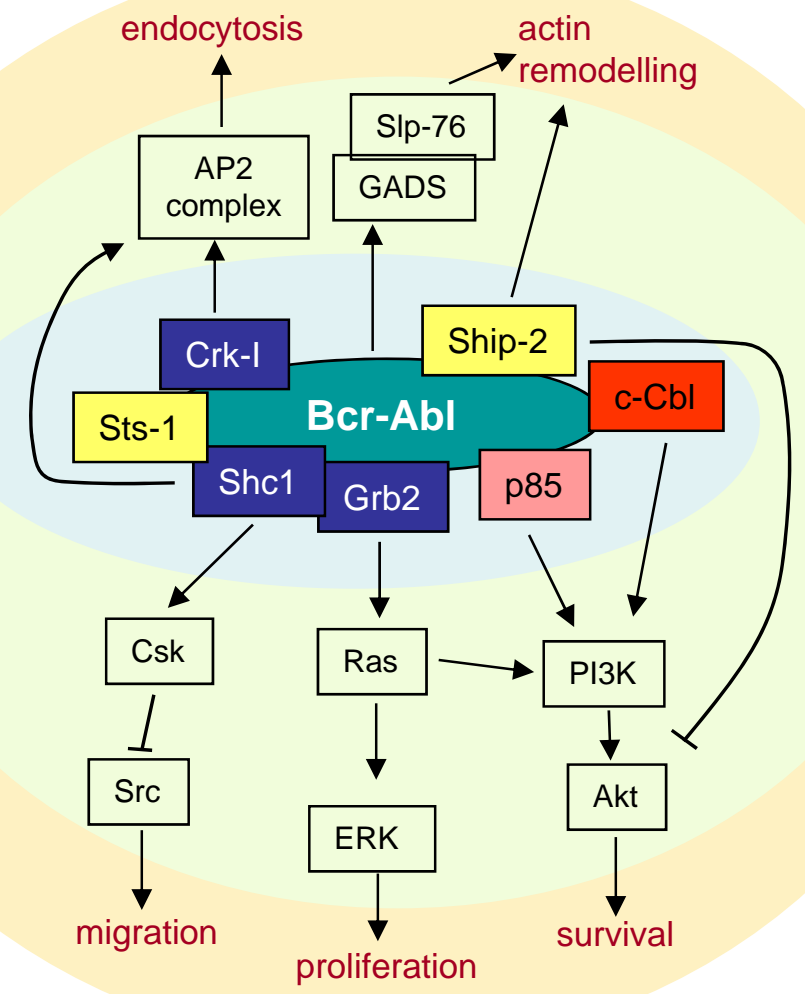


Fig. 3

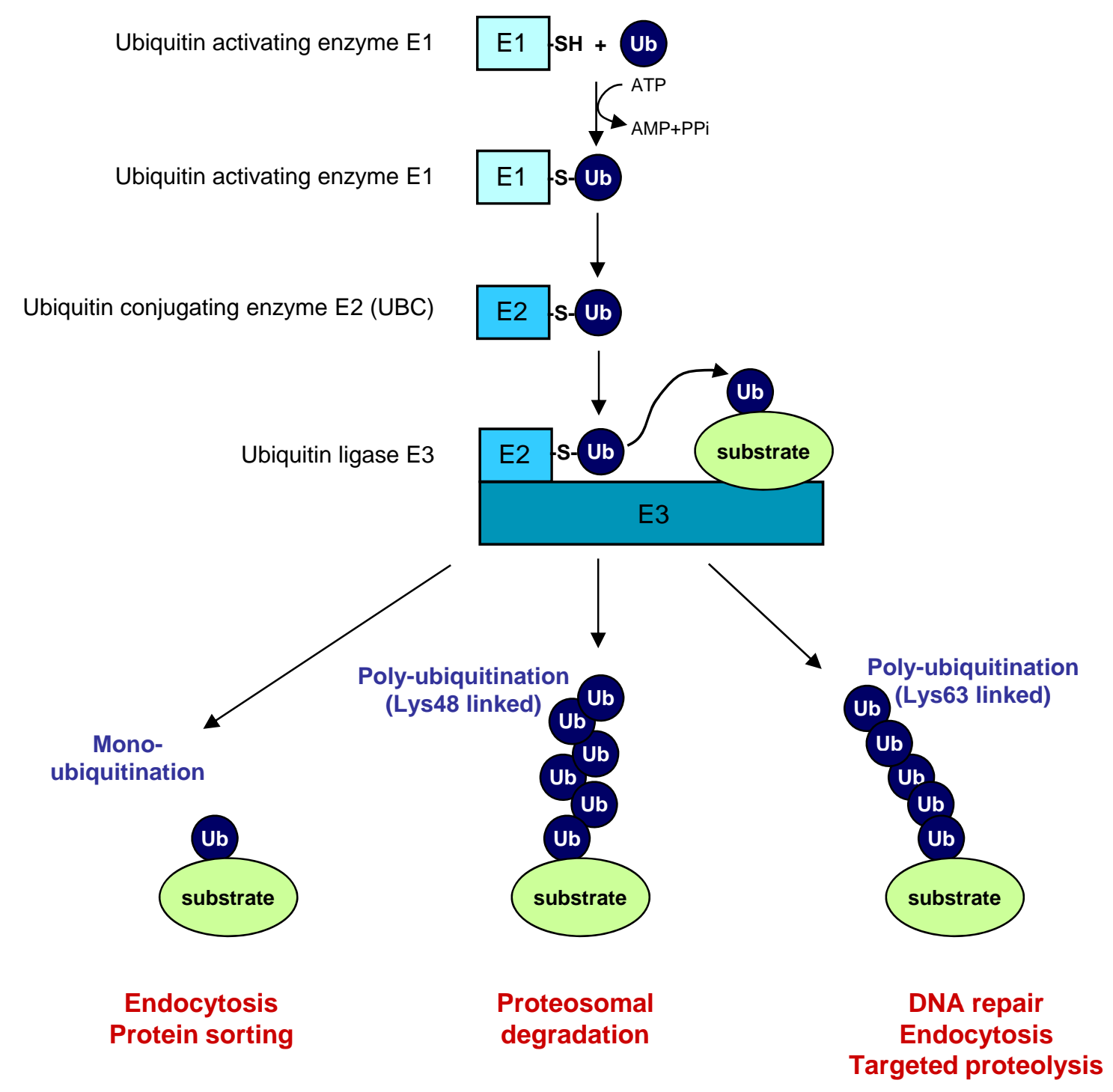

